\title{
Análisis espacial de la tasa de mortalidad infantil. Implementación de modelos de regresión espacial.
}

\author{
Spatial analysis of the infant mortality rate. Implementation of spatial \\ regression models.
}

Tulia Eva Salcedo Palacios. ${ }^{1}$, Andrés Felipe Ortiz Rico ${ }^{2}$

Recibido: 18-07-2017 / Revisado: 12-09-2018 Aceptado: 11-10-2018/ Publicado: 01-11-2018

\begin{abstract}
.
DOI: https://doi.org/10.33262/cienciadigital.v2i4.1..196

With the objective of being part of the Organization for Economic Cooperation and Development, OECD, Colombia has generated various public policies to reduce indicators of poverty, health, education, among others. One of the indicators that shows the quality of public health is the infant mortality rate, that is why some strategies have been strengthened in favor of its reduction; however, it is considered important to evaluate social inequality as an explanatory variable of the infant mortality rate in Colombia. In this research, a spatial analysis of the infant mortality rate in Colombia was carried out for 2016, in which it is sought to determine if there is spatial dependence, taking as delimited regions the municipalities of Colombia, this relationship is analyzed through the global and local Moran Index , finding clusters that determine spatial dependence at the municipal level. Finally, we estimate local and geographically weighted polynomial regression models using as covariables the prematurity rate, percentage of affiliates to the health regime and the Multidimensional Poverty Index. The implementation of these models managed to determine marked differences at the municipal level, which allows the recognition of high-risk areas; It is possible to generate programs that allow the reduction of infant mortality in these areas.
\end{abstract}

Keywords: Spatial analysis, infant mortality, spatial regression model.

\footnotetext{
${ }^{1}$ Universidad Santo Tomás, Departamento de. Ciencias Económicas y Administrativas, Facultad de Estadística, Bogotá, Colombia, tuliasalcedo@usantotomas.edu.co

${ }^{2}$ Universidad Santo Tomás, Departamento de. Ciencias Económicas y Administrativas, Facultad de Estadística, Bogotá, Colombia, andresortiz@usantotomas.edu.co
} 


\section{Resumen.}

Con el objetivo de hacer parte de la Organización para la Cooperación y el Desarrollo Económico, OCDE, Colombia ha generado diversas políticas públicas para reducir indicadores de pobreza, salud, educación, entre otros. Uno de los indicadores que evidencia calidad de la salud pública, es la tasa de mortalidad infantil, es por esto que se han fortalecido algunas estrategias en pro de su reducción, sin embargo, se considera importante a su vez evaluar la desigualdad social como variable explicativa de la tasa de mortalidad infantil en Colombia. En esta investigación se realizó un análisis espacial de dicha tasa en Colombia para el año 2016, en la cual se busca determinar si existe dependencia espacial, tomando como regiones delimitadas los municipios de Colombia, dicha relación es analizada mediante el Índice de Morán global y local, encontrando clúster que determinan dependencia espacial a nivel municipal. Finalmente se estiman modelos de regresión polinomial local y ponderado geográficamente usando como covariables la tasa de prematuridad, porcentaje de afiliados al régimen de salud y el Índice de Pobreza Multidimensional. La implementación de estos modelos logró determinar diferencias marcadas a nivel municipal lo que permite reconocer zonas de alto riesgo; pudiéndose generar programas que permitan la reducción de mortalidad infantil en dichas zonas.

Palabras Claves: Análisis espacial, mortalidad infantil, modelo de regresión espacial.

\section{Introducción.}

La tasa de mortalidad infantil (TMI) se valora como el cociente entre el número de infantes menores de un año sobre el total de nacidos vivos en este mismo periodo. El correcto cálculo de ésta permite al Estado determinar de manera asertiva proyectos de ley que garanticen suplir las necesidades de la población a nivel social y económico. En los países y regiones de menor desarrollo económico los registros de muertes son inexistentes o poco confiables (Ordoñez \& Prieto, 2017), esto debido a los diferentes subregistros que se presentan a nivel hospitalario de nacidos vivos y muertes en el primer año de vida. Entre tanto, la mortalidad infantil (MI) se ha considerado como uno de los principales indicadores de desarrollo social y económico; específicamente, es un indicador del estado de salud general de la población (Castillo \& Arocha, 2017), es por esto que en los últimos años el país ha tenido como objetivo gubernamental reducir la MI, con el fin de generar indicadores de salud pública más favorables. Para lograr lo anterior, se vienen desarrollando diversas políticas y estrategias en torno al tema; los avances obtenidos han sido mejorar la salud infantil en las diferentes regiones del país y crear programas de educación en salud a mujeres en estado de embarazo; sin embargo, en los últimos años se ha hecho más notorio las brechas entre algunas regiones más vulnerables versus otras que no están consideradas con esta condición (Mogollón, 2016). Como solución a los problemas relacionados con el registro de las estadísticas vitales se han 
presentado diversas metodologías para analizar la TMI, en esta investigación se buscó abordar este importante tema haciendo implementación del análisis espacial referente a datos de áreas y empleando como covariables explicativas la tasa de prematuridad (TP), el porcentaje de afiliados al sistema de salud y el Índice de Pobreza Multidimensional (IPM), éste último como indicador estadístico que revela desarrollo social y mide las privaciones humanas.

Las causas de la mortalidad infantil son en su mayoría evitables. En Colombia, las más frecuentes están asociadas a la prematurez, la presencia de anomalías congénitas e infecciones neonatales (Jaramillo, 2016), todas éstas son previsibles si se cuenta con educación y controles prenatales efectivos, además del acceso a un sistema de salud preparado para resolver cualquier complicación posparto. La prematuridad es una condición frecuente y es responsable de elevadas tasas de morbilidad y mortalidad, afectando de forma significativa los periodos gestacionales de mujeres que se hallan en desventaja social y económica (Ministerio de Salud y Protección Social., 2013). Así mismo, el peso al nacer es uno de los mejores predictores de la mortalidad infantil (Castro, Salas, Acosta, Delgado \& Calvache, 2016). En Colombia, de acuerdo a estadísticas del Departamento Administrativo Nacional de Encuestas (DANE), para el año 2015, la tasa del bajo peso al nacer no se ha modificado y se mantiene alrededor del $9 \%$ y la prematuridad es del $19.3 \%$.

Un ejemplo contrario a lo anteriormente descrito es Cuba, país que posee la tasa de mortalidad infantil más baja en Latinoamérica. Ésta está asociada al desarrollo social con prioridad de la salud y la educación, la implantación de un sistema nacional de salud que da cobertura al total a la población, un gran desarrollo de la atención primaria y la participación de la población mediante sus organismos en las tareas sanitarias (Lurán, Pinilla \& Sierra, 2009). Estas condiciones favorables garantizan que el transcurso del embarazo en las mujeres tenga un seguimiento efectivo y que la mayor parte de los embarazos llegue a buen término, aspectos que son de relevancia para el análisis generado en la presente investigación.

Durante la audiencia pública de rendición de cuentas de 2015, el Ministro de Salud y Protección Social, Alejandro Gaviria Uribe, precisó que en consecuencia, la población cubierta al sistema de salud llegaba a la cobertura del $97.6 \%$, los cuales el $44.5 \%$ de la población total se encuentra afiliada al régimen contributivo, el $48.1 \%$ al régimen subsidiado y el 5\% a los regímenes especiales y de excepción(Ministerio de Salud y Protección Social., 2015), no obstante, los resultados del sistema actual han revelado una persistente dificultad para que las pacientes de escasos recursos económicos tengan un acceso expedito a los servicios de salud con calidad, en su defecto hay un deficiente servicio y un aumento en su costo total (Ortíz, Navarro \& Eslava, 2013), además, aún se observa como día a día el acceso a la educación, a la alimentación y a los sistemas de salud de la población infantil es inequitativo y pareciese que la sobrevida de este grupo etario vulnerable, depende de la zona geográfica del país donde nace y vive (Restrepo, 2017). 
La escasa equidad en el acceso de las mujeres y los niños a servicios de salud de calidad, hace evidente la diferencia tan significativa a nivel municipal; la solución no se encuentra en sólo disminuir la mortalidad infantil a nivel general, sino además a nivel municipal y garantizar un sistema de salud digno e incluyente. Con base a lo anterior, este estudio busca encontrar la relación espacial para describir el comportamiento que registra la TMI con respecto a la tasa de prematuridad (TP), proporción de habitantes del municipio afiliados a salud y el índice de pobreza multidimensional (IPM). El anterior análisis permite visualizar una dinámica a nivel municipal que sugiere relaciones de la MI con aspectos de índole social, lo que al futuro permitirá desarrollar políticas públicas más efectivas y que logren mejorar de manera sustancial la salud de las poblaciones más vulnerables.

En el presente artículo se realiza un análisis espacial de datos de área para determinar un patrón espacial e identificar agregaciones espaciales para la TMI a nivel municipal en Colombia para el año 2016. Se realiza un análisis exploratorio de los datos para describir y visualizar las distribuciones espaciales de la TMI, seguido se realiza un análisis de autocorrelación espacial para determinar en que zonas se presentan agrupaciones espaciales y finalmente un análisis confirmatorio de los datos mediante modelos de regresión ponderados geográficamente empleando como variables regresoras algunos indicadores de salud pública: la tasa de prematurez, porcentaje de afiliados a los regímenes de salud existentes y el Índice de Pobreza Multidimensional.

\section{Materiales y métodos .}

Se realizó un análisis espacial de datos de área para la TMI en Colombia para el año 2016, delimitando las regiones analizadas por los 1122 municipios de Colombia implementado la utilización de software de sistemas de información geográfica (SIG) de uso libre QGIS. Se determinan clúster con mayor afectación de la TMI y se proponen tres modelos de regresión polinómica local y ponderada geográficamente usando como variables regresoras como la tasa de prematuridad, porcentaje de afiliados al régimen de salud y el Índice de Pobreza Multidimensional (IPM), dichos análisis se realizaron mediante el uso del software SIG GeoDa.

\section{Datos.}

La mayoría de las muertes en menores de cinco años se han considerado como inequitativas, puesto que reúnen características evitables e inadecuadas. Así mismo, la tasa de mortalidad infantil es un indicador que refleja las condiciones de salud y desarrollo de los países, y evidencia el nivel de prioridad que dan los gobiernos al derecho a la salud (Ministerio de Salud y Protección Social, 2016).

Se emplea para el análisis de esta investigación información de defunciones correspondientes a la consolidación, codificación de causas de muerte, validación y procesamiento realizado 
por el DANE, según las Estadísticas Vitales, a partir de los certificados de defunción físicos o digitales diligenciados por médicos para el año 2016 (DANE, 2017). Se determina la tasa de mortalidad infantil, como el número de defunciones de menores de cuatro años durante 2016 sobre el número de nacidos vivos en el mismo año y se representa por cada 1000 habitantes.

Un neonato se considera prematuro si ha nacido vivo antes de se hayan cumplido las 37 semanas de gestación.

Las complicaciones relacionadas con la prematuridad son la principal causa de defunción en los niños menores de cinco años. Para el 2015 se generaron aproximadamente un millón de muertes a nivel mundial, se calcula que tres cuartas partes de éstas son evitables si se cuenta con un sistema de salud capaz de subsanar de manera inmediata las complicaciones de estos partos (Organización Mundial de la Salud, 2018).

Para el análisis de esta variable se tomó a consideración las estadísticas vitales del número de nacimientos fetales menores a 37 semanas del año 2016 con respecto del número total de nacimientos a término de este año y se estandariza por cada 1000 habitantes.

Por otra parte, se emplea el número de afiliados al sistema de salud para el año 2016, sea régimen subsidiado o contributivo, respecto de la proyección municipal realizada por el DANE, los datos sobre el total de afiliados se encuentra en datos abiertos del Ministerio de Salud. Finalmente se aplica el Índice de Pobreza Multidimensional, desarrollado en la Universidad de Oxford, que utiliza microdatos de encuestas de hogares para su cálculo, donde cada miembro de una familia es clasificado como pobre o no pobre dependiendo de 5 factores analizados: vivienda, salud, educación, trabajo, juventud y niñez.

El IPM surge como respuesta a la necesidad de contar con una medida de pobreza con el propósito de hacer un diseño y seguimiento a la política pública.

De igual manera, la posibilidad de realizar un análisis profundo de múltiples dimensiones de la pobreza diferente al ingreso, hizo del IPM un instrumento útil para reflejar las privaciones que debe enfrentar un hogar en Colombia, y por ende ideal para el seguimiento de la normativa actual (DANE, 2017).

Este indicador nos permitió realizar comparaciones a nivel municipal, con el fin de medir carencias básicas en las distintas poblaciones analizadas en esta investigación.

El análisis exploratorio de datos espaciales se realizó con el sistema de información geográfica libre y de código abierto QGIS que permitió visualizar la información geoespacial de la TMI, mientras que el análisis de autocorrelación espacial global y local, al igual que el modelamiento espacial propuesto con las variables regresoras se realizó con el software libre GeoDa desarrollado por el Dr. Luc Anselin y su equipo. 
Tanto QGIS como GeoDa ofrecen al investigador un programa con una interfaz gráfica fácil y práctica para realizar análisis de datos espaciales.

\section{Análisis espacial.}

La estadística espacial se divide en tres áreas, la implementación adecuada de cada una de ellas depende exclusivamente de las características de la información que se desea analizar, para los datos regionales que se quieren describir en este artículo es pertinente realizar un análisis basado en datos de áreas; ya que es un proceso estocástico con un espacio de parámetros discretos donde se tiene una selección de los sitios de medición, es decir los municipios de Colombia, éstas unidades de muestreo pueden ser o no igualmente espaciadas.

El análisis estadístico de datos de área busca como objetivo la determinación de una estructura de autocorrelación espacial. La noción de autocorrelación espacial de estas variables está asociada con la idea de que valores observados en áreas geográficas adyacentes serán más similares que los esperados bajo el supuesto de independencia espacial (Giraldo, Caballero \& Camacho, 2018).

Para lo anterior se analizó con la proximidad Queen, con lo cual se determinó tener en cuenta todos los vecinos adyacentes para el análisis de la autocorrelación espacial. Una herramienta que define esta dependencia espacial es el Índice de Moran (IM) que permite definir la proximidad entre áreas y para el estudio local de estas agrupaciones se aplicó el Índice Local de autocorrelación Espacial (LISA), lo anterior se lleva a cabo por medio del cálculo de una matriz de proximidad espacial que son una función de la matriz de datos originales obtenidos por la proximidad de tipo Queen de donde dado un conjunto de áreas $\left(A_{1}, A_{2}, \ldots, A_{n}\right)$ se construye una matriz $W^{(1)}{ }_{n x n}$ donde cada elemento $w_{i j}$ representa la proximidad de las áreas $i, j$.

Índice de Morán: En general, este índice se interpreta similar al coeficiente de correlación r empleado en modelos lineales, se acepta que existe autocorrelación espacial (AE) siempre que haya una variación espacial sistemática en los valores de una variable a través de un mapa, es decir un patrón en el comportamiento de la variable según la ubicación geográfica del dato. Sean $\left(z_{1}, z_{2}, \ldots, z_{n}\right)$ variables aleatorias medidas en $n$ áreas. Existirá correlación espacial si los valores observados en áreas geográficas adyacentes serán similares a los esperados bajo el supuesto de independencia espacial. Para medir esta correlación se puede utilizar el índice de Moran definido como:

$$
I=\frac{n \sum_{i=1}^{n} \sum_{j=1}^{n} w_{i j}\left(z_{i}-\bar{z}\right)\left(z_{j}-\bar{z}\right)}{\sum_{i=1}^{n} w_{i} \sum_{i=1}^{n}\left(z_{i}-\bar{z}\right)^{2}}
$$


Donde $w_{i j}$ representa la distancia entre dos centros si $A_{i}$ comparte frontera con $A_{j}$ entonces $w_{i j}$ será 1 , en otro caso será 0 . Si los valores altos de una localización están asociados con valores altos en los vecinos, la autocorrelación espacial es positiva siendo la situación opuesta la de autocorrelación espacial negativa (Cepeda \& Velásquez, 2004), para este estadístico es importante probar la hipótesis:

$$
H_{0}: I=0 \quad \text { vs } \quad H_{a}: I \neq 0
$$

Bajo el supuesto de normalidad se tienen los siguientes resultados:

$$
E(I)=-\frac{1}{n-1} \quad \text { y } \quad \operatorname{Var}(I)=\frac{\left(n^{3}-n^{2}\right) S_{1}+\left(n-n^{2}\right) S_{2}+(2-4 n) S_{0}^{2}}{\left(n^{3}-n^{2}-n+1\right) S_{0}^{2}}
$$

Donde

$$
S_{0}=\frac{1}{2} \sum_{i=1}^{n} \sum_{j=1}^{n} w_{i j} \quad, \quad S_{1}=\frac{1}{2} \sum_{i=1}^{n} \sum_{j=1}^{n}\left(w_{i j}+w_{j i}\right)^{2} \quad \mathrm{y} \quad S_{2}=\sum_{i=1}^{n} \sum_{j=1}^{n}\left(w_{i .}+w_{. j}\right)^{2}
$$

Con lo cual el estadístico $\frac{I-E(I)}{\sqrt{\operatorname{Var(I)}}}$ sigue una distribución normal estándar.

También es posible utilizar una prueba de permutaciones para verificar la hipótesis de interés probando la significancia del resultado obtenido de la siguiente manera:

- Se calcula el estadístico original $I_{0}$.

- Se construye una permutación de los datos originales, un intercambio entre las áreas y los valores observados $z_{i}$.

- Se calcula el índice sobre la permutación $I_{p}$.

- Se repiten los pasos 2 y 3 y se estima el valor p de la prueba como el número de $I_{p}>$ Io sobre el número de permutaciones.

Los procedimientos empleados para evaluar la significación estadística consisten en una simulación de Monte Carlo de diferentes arreglos de los datos y la construcción de una distribución empírica de estadísticas simuladas. Posteriormente, el valor obtenido originalmente se compara con la distribución de los valores simulados y si el valor supera el percentil 95, se dice que la relación encontrada es significativa al 5\%. 
Sin embargo, cuando se tiene un gran número de áreas, en esta investigación se emplearon 1122 áreas, es posible que existan diferencias en la asociación espacial. Para hacer frente a esas diferencias espaciales se utilizó el Índice Local de Moran, conocido como el Índice Local de Autocorrelación Espacial (LISA) (Anselin, 1995).

Índice Local de Autocorrelación Espacial (LISA): Se propone un conjunto de indicadores locales de asociación espacial, LISA ("Local Indicators of Spatial Association"), capaces de detectar la contribución de cada región a un indicador de dependencia espacial global (Anselin, Florax \& Rey, 2013), los indicadores LISA presentados para este trabajo serán basados en I de Morán. El índice local de Morán se expresa como:

$$
I_{i}=\frac{\left(z_{i}-\bar{z}\right)}{S_{i}^{2}} \sum_{i=1}^{n} w_{i j}\left(z_{j}-\bar{z}\right)
$$

Donde

$$
S_{i}^{2}=\frac{\sum_{\substack{j=1 \\ i \neq j}}^{n} w_{i j}}{n-1}-\bar{z}^{2}
$$

Su valor esperado y varianza están dados por:

$$
E\left(I_{i}\right)=-\frac{\sum_{j=1}^{n} w_{i j}}{n-1} \quad \mathrm{y} \quad \operatorname{Var}\left(I_{i}\right)=\frac{w_{i(2)}\left(n-b_{2}\right)}{n-1}+2 w_{i(k h)}
$$

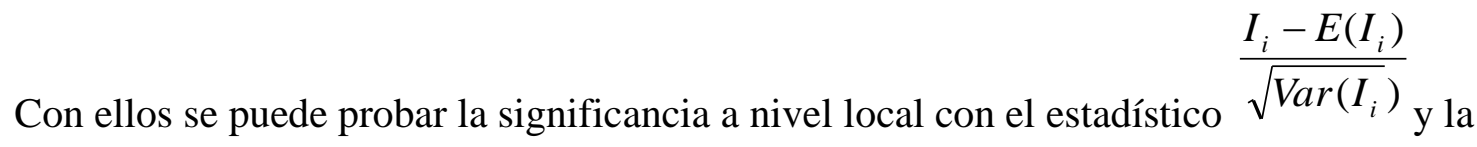
distribución normal estándar.

Modelos de regresión espacial: La primera idea para modelar datos espaciales consiste en incluir como covariables de un modelo que tenga en cuenta sólo datos de georreferenciación, empero esta técnica no tiene en cuenta el valor de las áreas vecinas. Una forma de explorar la variación espacial es a partir del cálculo de la variable rezagada espacialmente, la cual se construye como un promedio ponderado del valor de la variable en los vecinos: 


$$
z_{i}^{*}=\frac{1}{n} \sum_{i=1}^{n} w_{i j} z_{i}, \quad \operatorname{con} w_{i j}=1 \text { ó } \quad w_{i j}=0
$$

Un mapa de $z_{i}{ }^{*}$ permite tener menor variabilidad entre los valores mientras que un gráfico de dispersión entre $z_{i}$ y $z_{i}{ }^{*}$ permitirá analizar la dependencia espacial existente en los datos. Los modelos para datos espaciales pueden ser vistos como análogo a los modelos AR y MA en series de tiempo, estos incluyen como información auxiliar, la información de $\mathrm{Z}$ o de los residuales $\varepsilon$ en áreas vecinas. La forma general del modelo autoregresivo espacial SAR es:

$$
Z=X \beta+\lambda W(Y-X \beta)+\varepsilon
$$

Donde:

- $\quad W$ es la matriz de proximidad.

- $\lambda$ es el peso para el componente espacial (escalar).

- $\quad$ Si $\lambda=0$ no existe dependencia espacial.

Del modelo SAR se puede ver que:

$$
\begin{array}{r}
(Z-\lambda W Z-X \beta+\lambda W X \beta)=\varepsilon \\
(I-\lambda W)(Z-X \beta)=\varepsilon \\
(Z-X \beta)=(I-\lambda W)^{-1} \varepsilon \\
Z=X \beta+(I-\lambda W)^{-1} \varepsilon
\end{array}
$$

Se puede denotar de lo anterior que la $(I-\lambda W)$ tiene que ser invertible. Si $W$ es simétrica y está estandarizada por filas se garantiza la invertibilidad para todo $\lambda \in(0,1)$ Por lo tanto:

$$
E(Z)=X \beta \quad \text { y } \quad \operatorname{Var}(Z)=(I-\lambda W)^{-1} \operatorname{Var}(\varepsilon)(I-\lambda W)^{-1}
$$

Con lo cual el modelo puede ser visto como un modelo lineal con una estructura particular para la matriz de varianza covarianza, que considera la cercanía especial entre las áreas. Para la estimación de parámetros se pueden utilizar por máxima verosimilitud iterando de la siguiente forma:

1. Estimar $\beta$ por MCO del modelo $Z=X \beta+\varepsilon$

2. Estimar $\operatorname{Var}(\varepsilon)$ y $\lambda$ maximizando la verosimilitud $\operatorname{con} \beta$ del paso anterior 
3. Estimar $\beta$ por MCG $\hat{\beta}=\left(X^{\prime} \hat{\operatorname{Var}}(\varepsilon) X\right)^{-1} X^{\prime} \hat{\operatorname{Var}}(\varepsilon) Z$

4. Repetir 2 y 3 hasta obtener convergencia de $\hat{\beta}, \hat{\operatorname{Var}}(\varepsilon)_{\mathrm{y}} \hat{\lambda}$.

Para explicar la TMI en Colombia, se analizaron tres modelos: modelo clásico estimando los parámetros por mínimos cuadrados ordinarios (MCO), el modelo rezagado espacialmente, el cual asigna una estructura de autocorrelación a los residuales de un modelo de regresión lineal descrito así, estimando los parámetros por máxima verosimilitud:

$$
Z=\rho W Z+X \beta+\varepsilon
$$

Por último, se implementó un modelo lineal con errores correlacionados donde:

$$
\begin{aligned}
& Z=X \beta+v \\
& v=\lambda W_{v}+\varepsilon
\end{aligned}
$$

\section{Resultados.}

El método de clasificación para la creación de los mapas cloropéticos es de rupturas naturales, o método de pausas naturales y se emplea ya que busca reducir la varianza dentro de las clases y maximizar la varianza entre las mismas; es decir, para cada segmentación los datos son muy homogéneos dentro del grupo pero muy heterogéneo entre ellos.

Los municipios con mayor tasa de mortalidad infantil fueron San Juan del Cesar (La Guajira), Jericó (Antioquia), Yavaraté (Vaupés), Puerto Santander, Bojayá, Alto Baudó (Chocó), Pacoa (Amazonas) y California (Santander). Los municipios con la mayor tasa de mortalidad por prematuridad fueron: Abriaquí, Heliconia (Antioquia), Puerto Santander (Chocó), Pana Pana (Guainía), Palmar, San Cristóbal (Santander), Mirití Paraná (Amazonas) (Figura 1). 


\section{TASA DE MORTALIDAD INFANTIL (TMI) POR CADA 1000 HABITANTES}

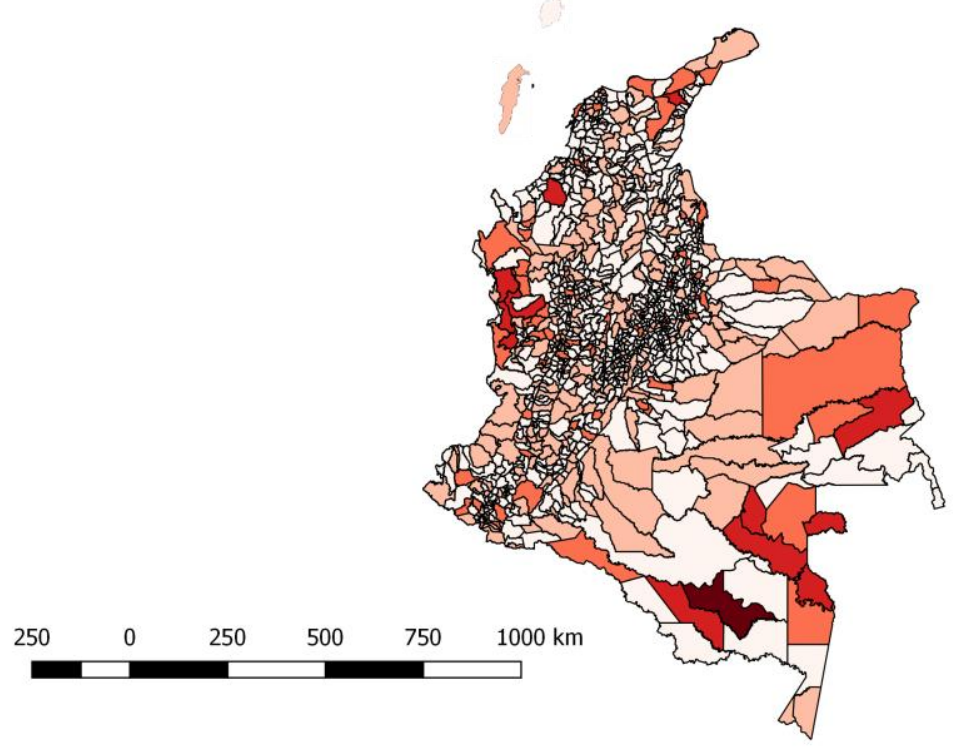

Defunciones no fetales

$\square 0.0000-5.1282$

$\square .1282-16.9492$

$\square 16.9492-36.4465$

$\square 36.4465-73.1707$

$73.1707-222.2222$

TASA DE PREMATURIDAD (TP) POR CADA 1000 HABITANTES

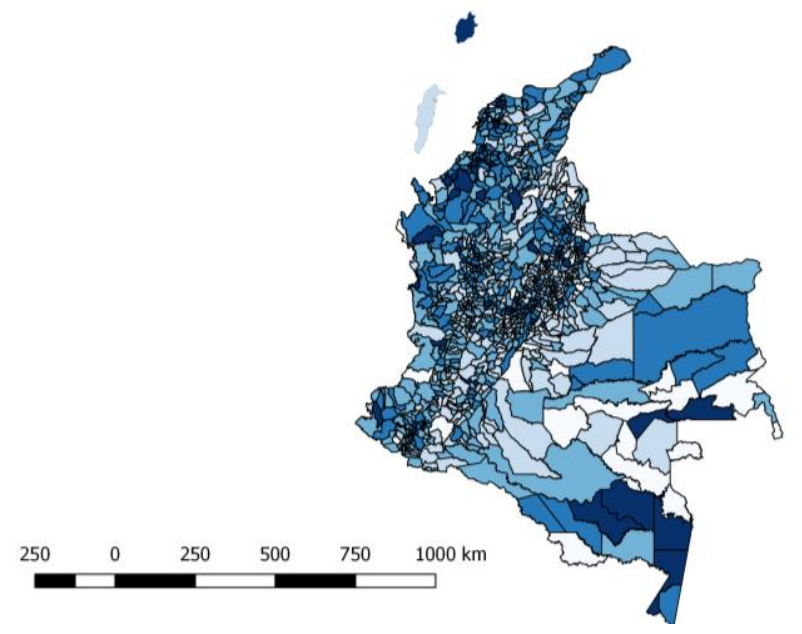

Nacimientos prematuros

$\square \quad 0.0$ - 93.8

$\square 93.8-156.7$

$156.7-204.3$

$204.3-263.2$

ש263.2- 500.0

Figura 1. Comparativo TMI y TP por municipio en Colombia para el año 2016.Fuente (DANE, 2017). Elaboración propia. 
Respecto de los habitantes afiliados a un sistema de salud se denota que en el departamento de Amazonas se evidencia un gran porcentaje de personas que no se encuentran inscritas a este servicio básico. Esto mismo aconteció en Guainía y parte del departamento de Vaupés (Figura 2), donde su mayoría de población son pueblos indígenas, la cual es considerada una población vulnerable en Colombia.

TASA DE AFILIADOS AL SISTEMA DE SALUD

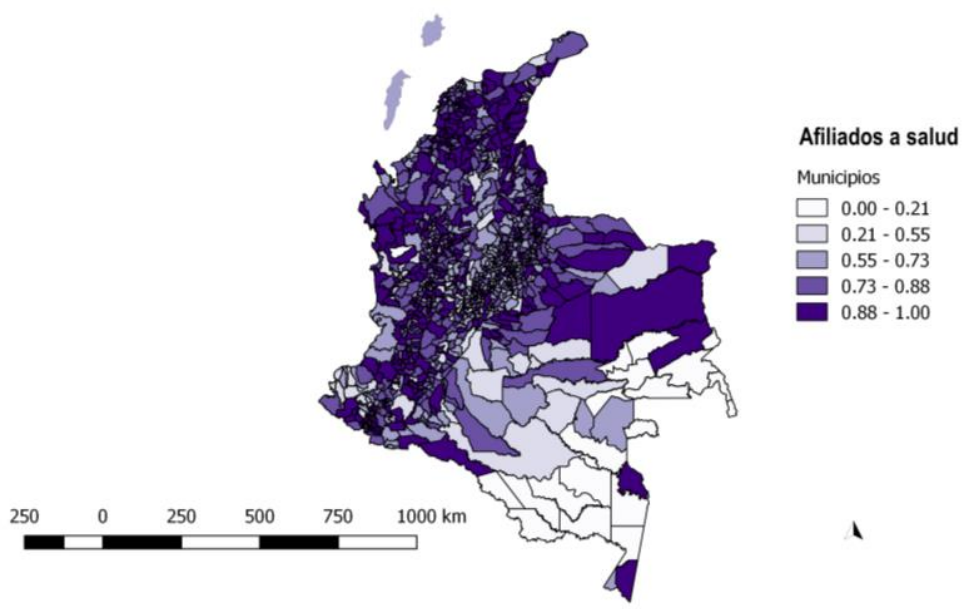

Figura 2. Porcentaje de afiliados al sistema de salud por municipio en Colombia para el año 2016. Fuente (Ministerio de Protección Social, 2017). Elaboración propia.

Para el análisis del IPM se denota que para el año tomado los índices de pobreza en Colombia eran muy altos, en particular, se identifica un elevado índice de pobreza en la zona de Nariño, Amazonas, Vichada, Chocó y Guajira, comparando esto con el mapa de mortalidad, se identifica una concentración similar (Figura 3), lo que vislumbra una idea del comportamiento de la TMI respecto de estas regiones. Aquellos municipios con índices cercanos a 1 indican niveles de pobreza altos, indicando que los habitantes de esto municipios no tienen forma de subsanar sus necesidades básicas o de primera necesidad. 


\section{ÍNDICE DE POBREZA MULTIDIMENSIONAL(IPM)}

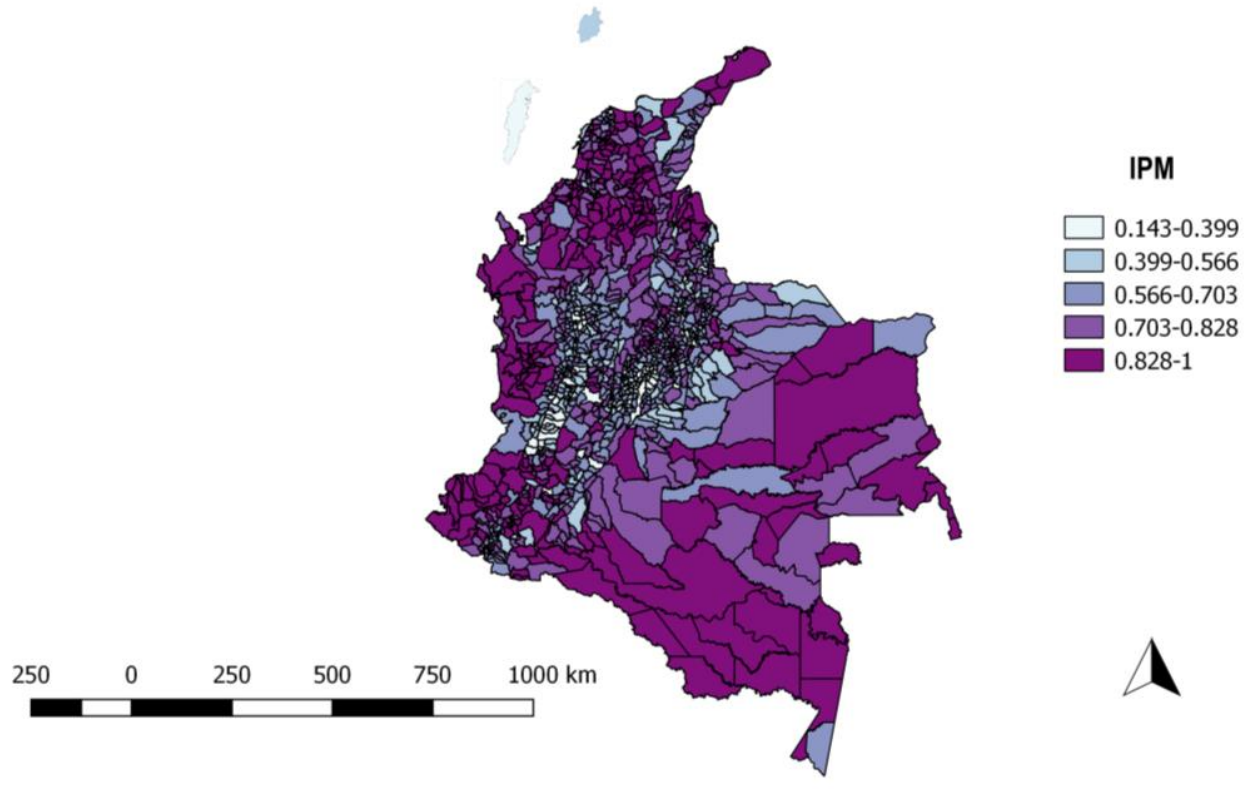

Figura 3. Índice de Pobreza Multidimensional por municipio en Colombia para el año 2016. Fuente (DANE, 2017). Elaboración propia.

Los índices de aleatoriedad espacial permiten relacionar la dependencia entre localizaciones. La estructura de esta aleatoriedad se compone de una matriz de pesos espaciales, que se puede calcular por diferentes métodos.

Se realizan cuadrantes y se determina en el caso de los ocho vecinos una contigüidad tipo Queen de primer orden donde se analiza la influencia que ejerce un punto $i$ sobre sus vecinos contiguos (Figura 4). Adicionalmente, se realizan las pruebas para el método de Rook de orden 1 y orden dos, el cual tiene en cuenta los cuadrantes en forma de cruz; los resultados obtenidos fueron más bajos por lo que se decide realizar el trabajo con el I de Morán basado en el método de Queen. 

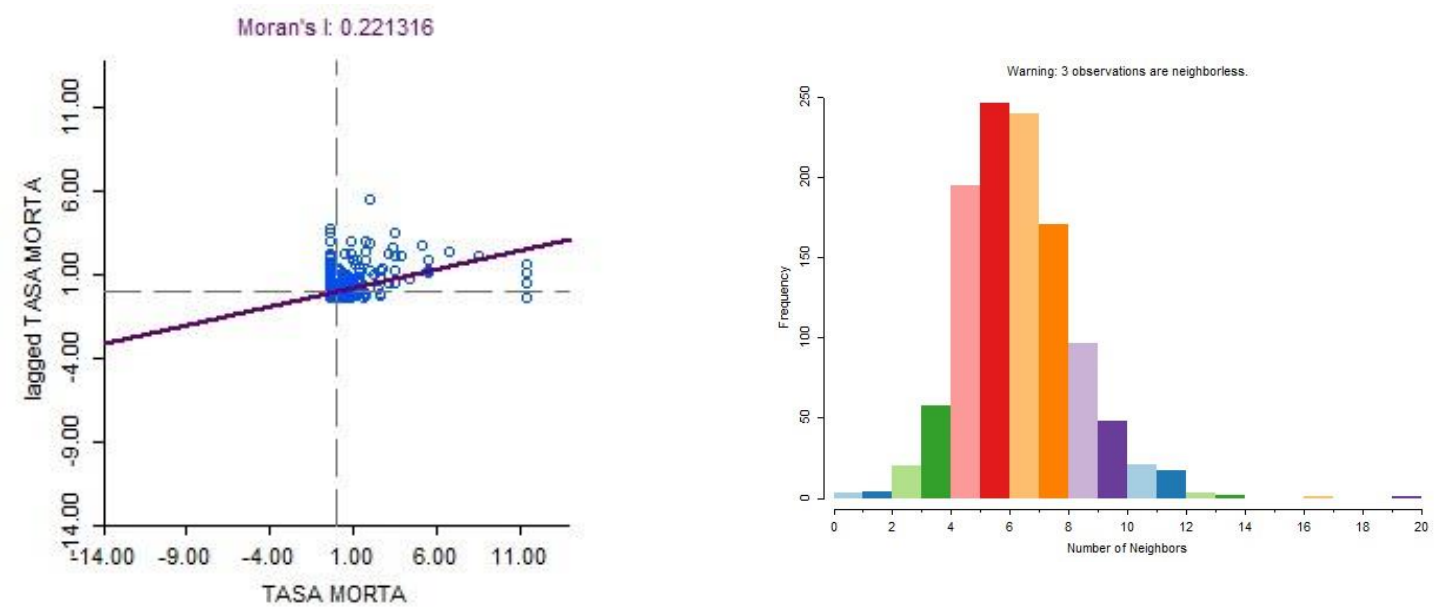

Figura 4. Índice de Pobreza Multidimensional por municipio en Colombia para el año 2016. Fuente (DANE, 2017). Elaboración propia.

El valor global del I de Morán y su valor esperado pueden ser calculados por medio de un proceso de aleatorización a través de permutaciones, la prueba se realizó con 999 de éstas, arrojando un valor de 0.221316 , además al realizarse la prueba de permutaciones, el pvalor fue de 0.001, por tanto se rechaza la hipótesis nula aceptando la alternativa (Figura 5). Existe autocorrelación espacial en la tasa de mortalidad infantil en Colombia para el año 2016.

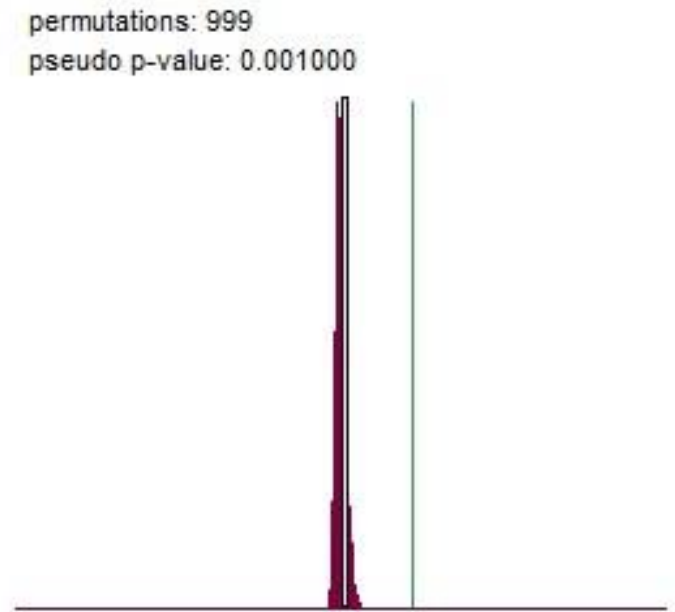

l: $0.2213 \mathrm{E}[\mathrm{]}$ : -0.0009 mean: -0.0011 sd: 0.0170 z-value: 13.0759

Figura 5. Prueba de permutaciones (999 permutaciones). Elaboración propia.

Una vez se haya seleccionado el método para determinar los pesos espaciales se realizó un análisis local a través de indicadores de asociación espacial local (LISA). Con este procedimiento se evalúa la hipótesis de aleatoriedad espacial comparando los valores del indicador (TMI) en cada municipio con los valores correspondientes de los municipios vecinos. Se observa que el departamento del Vichada, parte de Arauca, Vaupés, Amazonas, 
Chocó y Guajira presentan altas tasas de mortalidad infantil, exhibiendo un problema no sólo a nivel municipal sino a su vez departamental (Figura 6).
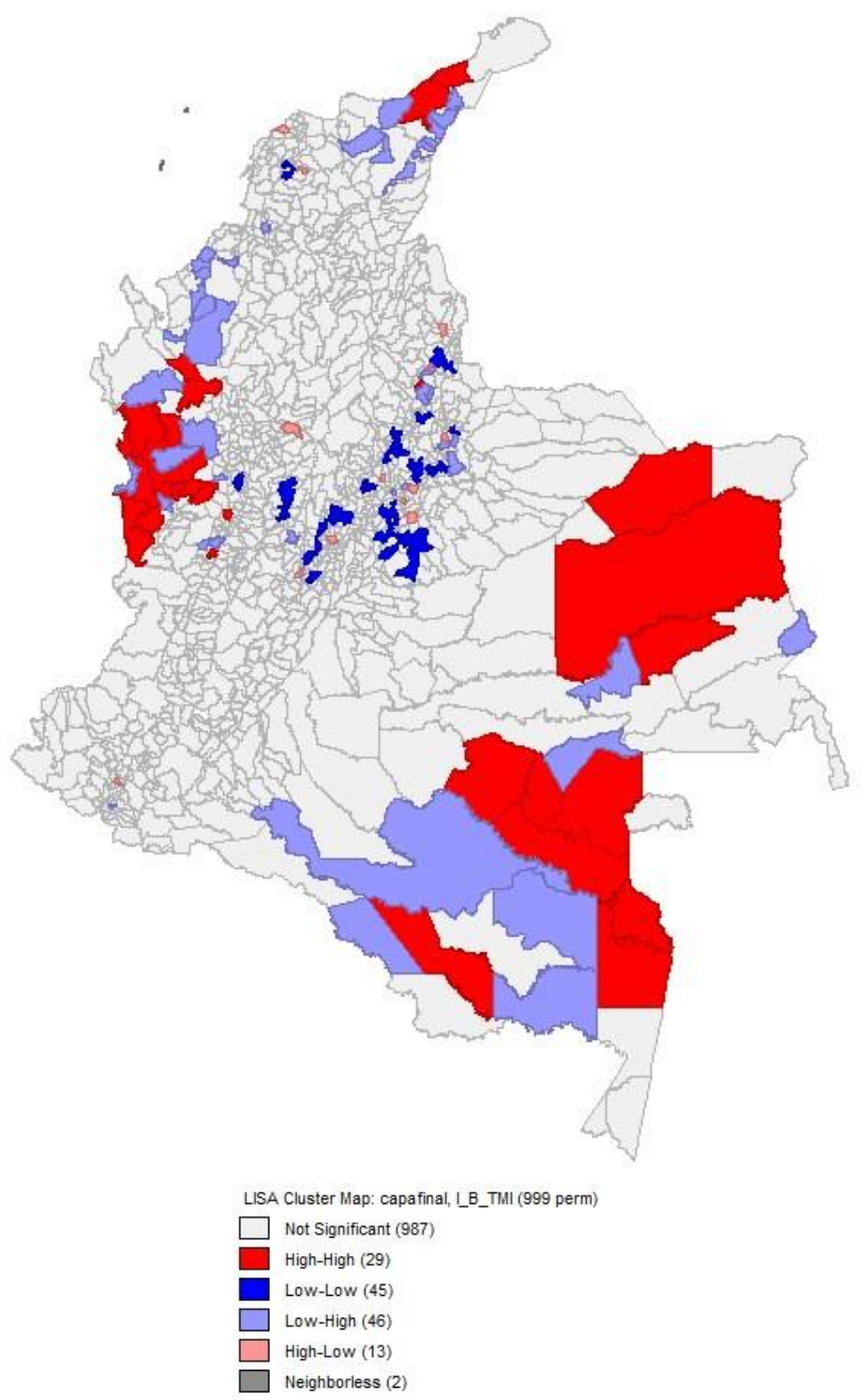

Figura 6. Mapa Clúster LISA para la TMI por municipio. Elaboración propia.

Se continuó explorando la información de las unidades espaciales por medios de los mapas de significancia, los cuales mostraron la probabilidad de que las relaciones de proximidad 
sean aleatorias. Por otra parte, se observa que la relación significativa se presenta en el sur y oriente del país en los departamentos de Amazonas, Vichada, Guaviare y parte de Arauca. Existen dos municipios que no pudieron ser analizados debido a las distancias que hay entre ellos y sus vecinos más cercanos, por lo que éste estudio no fue posible extenderlo a las islas de San Andrés y Providencia.

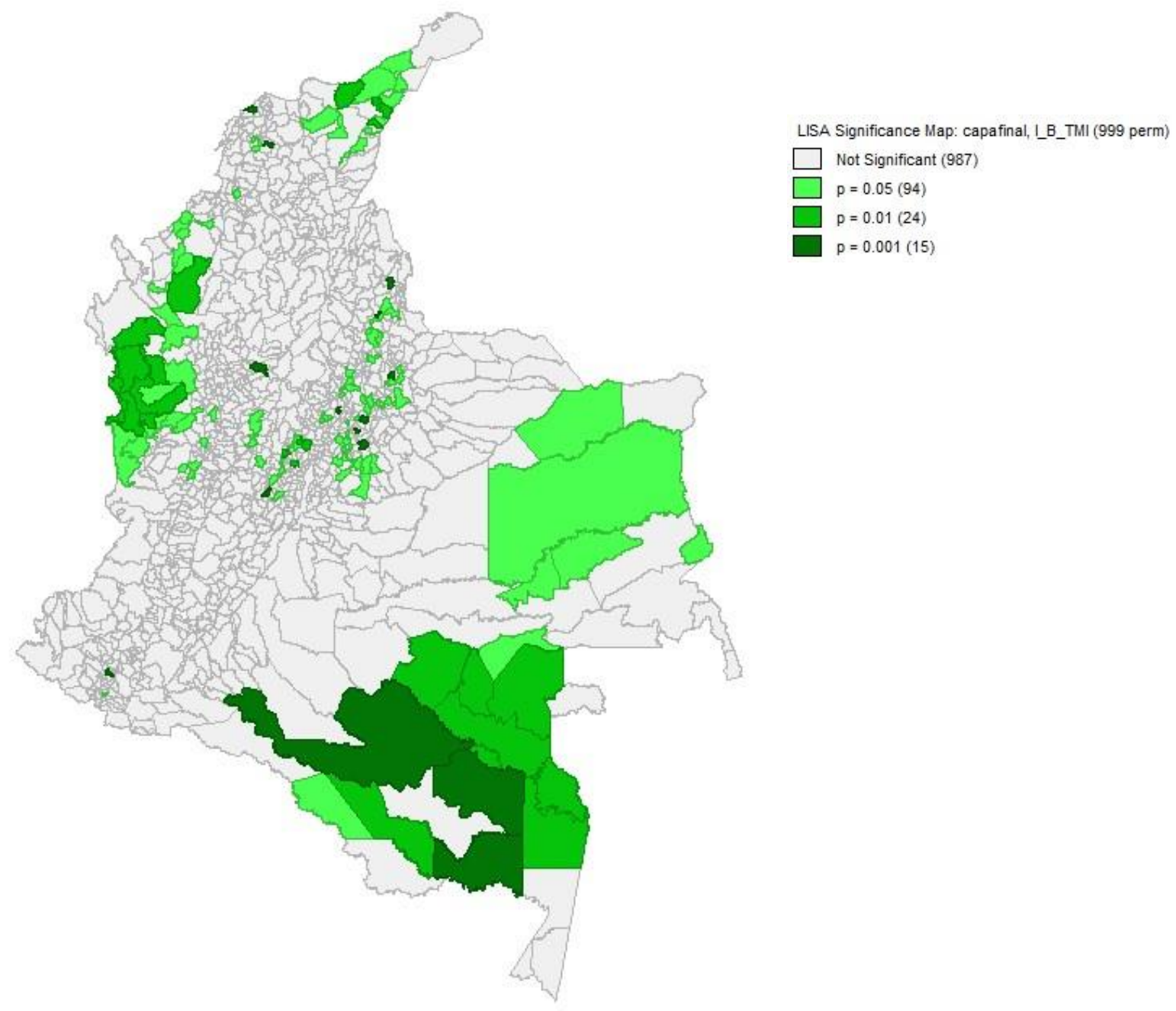

Figura 7. Mapa significancia LISA para la TMI por municipio. Elaboración propia.

La inferencia mediante modelos de regresión espacial es poco usada esto debido a que una proximidad espacial puede hacer que los elementos estudiados compartan características y esto haga que tengan comportamientos similares (Fang, Liu, Li, Sun, \& Miao 2015), aunque en recientes investigaciones sociales se ha implementado como una herramienta de análisis útil y clara, permitiendo visualizar fenómenos antes no descritos. En algunos modelos de regresión espacial es importante determinar si hay autocorrelación espacial en los residuos, por el supuesto de independencia y no correlación. Cuando los residuales se encuentran 
correlacionados es importante determinar las variables significativas del modelo y de ser posible existen modelos de regresión que permite modelar la autocorrelación espacial.

La técnica de regresión más conocida es Mínimos Cuadrados Ordinarios (OLS), permitirá crear una ecuación de regresión simple para representar la TMI en función de las variables aquí propuestas. Los resultados obtenidos por el método de mínimos cuadrados ordinarios (Tabla 1), mostraron un $r^{2}$ muy bajo (0.0074), el modelo da cuenta que la tasa de mortalidad aumenta conforme incrementa la tasa de prematuridad y el IPM, y a su vez el porcentaje de afiliados aumenta disminuye la TMI.

Tabla 1.Resultados modelo OLS.

\begin{tabular}{lccc}
\hline Variable & Coeficiente & Error estándar & Probabilidad \\
\hline Constante & 3.93 & 2.36 & 0.095 \\
B_IPM & 0.40 & 2.06 & 0.846 \\
B_SALUD & -2.24 & 1.73 & 0.195 \\
B_TP & 0.015 & 0.006 & 0.008 \\
\hline
\end{tabular}

Nota. Resultados obtenidos implementando el software GeoDa.

Se denota que hay autocorrelación espacial en los residuales por lo que se hace necesario estimar un modelo que controle este supuesto, una alternativa son los modelos de rezagado espacialmente (SAR) y los modelos de error espacial.

Para el modelo rezagado espacialmente se obtuvieron resultados similares, resaltando que los pesos cuentan con un coeficiente de 0.24 , determinando que es relevante el rol de la estructura de los vecinos cercanos, el $r^{2}$ es más alto de 0.04 y el AIC disminuyó a 8568 . La constante da positiva la cual es una observación a determinar en el modelo, se puede realizar sin constante, ya que no es significativa para ninguno de los modelos propuestos. (Tabla 2)

Tabla 2.Resultados modelo rezagado espacialmente (SAR).

\begin{tabular}{lccc}
\hline Variable & Coeficiente & Error estándar & Probabilidad \\
\hline W_B_TMI & 0.24 & 0.04 & 0.000 \\
Constante & 3.43 & 2.32 & 0.140 \\
B_IPM & -1.53 & 2.06 & 0.448 \\
B_SALUD & -1.13 & 1.70 & 0.507 \\
B_TP & 0.012 & 0.006 & 0.018 \\
\hline
\end{tabular}

Nota. Resultados obtenidos implementando el software GeoDa.

En el modelo del error espacial se denota un $r^{2}$ de 0.47 y unos coeficientes acordes a lo que se consideraba sucedería en el caso de mortalidad infantil aquí tratado. En este modelo la constante es positiva, pero al igual no significativa (Tabla 3). 
Tabla 3.Resultados modelo de error espacial.

\begin{tabular}{lccc}
\hline Variable & Coeficiente & Error estándar & Probabilidad \\
\hline Constante & 6.03 & 2.69 & 0.024 \\
B_IPM & -4.99 & 2.41 & 0.038 \\
B_SALUD & 0.33 & 1.84 & 0.859 \\
B_TP & 0.013 & 0.006 & 0.036 \\
LAMBDA & 0.282 & 0.043 & 0.000 \\
\hline
\end{tabular}

Nota. Resultados obtenidos implementando el software GeoDa.

\section{Discusión.}

Uno de los mayores problemas que se tiene en Colombia para determinar indicadores de mortalidad infantil es la estimación de la tasa a partir de las estadísticas vitales, es por esto que algunos autores (Ordoñez \& Prieto, 2017) proponen realizar una estimación indirecta de la misma. Por otra parte, (Ruiz \& Durán, 2013) propusieron el uso de estos registros para realizar un análisis temporal y determinaron mediante un estudio geoestadístico una estimación de dicha tasa. En esta investigación se buscará determinar si es posible explicar la tasa de mortalidad infantil en Colombia por aquellos neonatos que fallecen a causas de bajo peso al nacer, la desatención a nivel salud por municipio y la poca accesibilidad a la satisfacción de las necesidades básicas de la población.

Se evidencia una alta tasa de mortalidad infantil en los departamentos de La Guajira, algún área de los Llanos Orientales, parte del Chocó y Putumayo; departamentos donde históricamente se han presentado las tasas más altas de mortalidad infantil. Desde el análisis exploratorio se puede denotar un patrón geográfico similar para la tasa de prematuridad. Una de las complicaciones presentes en esta investigación, es que en diversas poblaciones de Colombia no se lleva un registro sistemático de los nacimientos, pues en general, los niños de hogares más pobres, rurales y cuyas madres tienen un bajo nivel de escolaridad enfrentan mayores riesgos de no contar con su partida de nacimiento (Bernal \& Forero, 2011). Por otra parte, en algunas poblaciones rurales o con comunidades indígenas se realizan partos en un ámbito extrahospitalario, por lo que no se registra el fallecimiento del neonato, ni se determina su causa de muerte. Adicionalmente, para algunos municipios se encontró que habían más afiliados al sistema de salud que habitantes, así como también se logró evidenciar que hay municipios que presentan un porcentaje nulo de afiliación, es decir, ningún habitante del municipio se encuentra registrado a cualquiera de los dos regímenes de salud en Colombia. Dada la relevancia del tema estudiado, se espera que los datos de la TMI en un futuro Colombia sean tratados con toda la tecnicidad posible, a fin de determinar políticas directas que minimicen en lo posible las muertes de niños durante los primeros años de vida. Una de las opciones son los modelos de análisis de tipo espacial aquí analizados que evidencian las regiones donde se focaliza con más intensidad esta problemática. 
La metodología aplicada del Índice de Morán midió la autocorrelación global de los municipios de Colombia y el comportamiento de la TMI respecto de las áreas vecinas; el resultado obtenido señala que la autocorrelación fue positiva pero no significativa, por tanto fue necesario estimar los clúster espaciales empleando el método LISA, calculado con 999 permutaciones el proceso de aleatorización y una matriz de pesos de proximidad espacial calculados por la metodología Queen. El mapa de significancia determinó que es significativa la relación espacial para la mayoría del territorio de los departamentos de Amazonas, Vichada, Chocó y La Guajira con un nivel de significancia menor a 0.05, para el caso especial de Amazonas y Vichada donde el p valor de la prueba es muy bajo. En este caso, existen estudios recientes que sugieren que la dinámica social, económica, ambiental, cultural, política y epidemiológica de los municipios fronterizos es diferente y que dichos aspectos pueden tener un impacto importante en la MI (Mogollón,2016).

Las variables regresoras, prematurez, IPM y afiliados al sistema de salud, se determinaron bajo el estudio retrospectivo de corte trasversal (Jaramillo, 2016), que empleó estadísticas vitales e información de registros de afiliación al sistema de salud. Lo anterior, permitió inferir que dichas variables influían en la tasa de mortalidad infantil; para las variables biológicas, el peso al nacer es un factor determinante en la supervivencia del niño haciendo de la prematurez la principal causa de mortalidad infantil y finalmente el factor socioeconómico tiene una influencia en la TMI, el cual se midió con el IPM por ser más exigente y determinar mejor la calidad de vida frente al Índice de Desarrollo Humano (IDH), el cual únicamente mide el ingreso que recibe cada individuo. En esta investigación se encontró bajo el modelo de error espacial que hay una relación espacial de tipo rezagado espacialmente entre estas covariables, ya que presenta el mejor $r^{2}$ de los tres modelos propuestos.

En Colombia, la falta de equidad en el acceso de las mujeres y los niños a servicios de salud de calidad implica la diferencia tan marcada a nivel municipal, la solución se encuentra en no sólo disminuir la mortalidad infantil a nivel general, sino además a nivel municipal garantizar un sistema de salud digna e incluyente que permita en estas regiones identificadas como de alto riesgo, focalizar programas que permitan la reducción de la mortalidad infantil.

\section{Referencias bibliográficas.}

Anselin, L., Florax, R., \& Rey, S. J. (Eds.). (2013). Advances in spatial econometrics: methodology, tools and applications. Springer Science \& Business Media.

Anselin, L. (1995). Local indicators of spatial association-LISA. Geographical analysis; 27(2), 93-115.

Bernal-Acevedo, O., \& Forero-Camacho, J. C. (2011). Sistemas de información en el sector 
salud en Colombia. Revista Gerencia y Políticas de Salud, 10(21).

Castillo Guzmán, A., \& Arocha Mariño, C. (2017). La salud pública en Cuba a pesar de la crisis económica internacional. Infodir (Revista de Información para la Dirección en Salud), 13(24), 100-109.

Castro-Delgado, Ó. E., Salas-Delgado, Í., Acosta-Argoty, F. A., Delgado-Noguera, M., \& Calvache, J. A. (2016). Muy bajo y extremo bajo peso al nacer. Pediatría, 49(1), 23-30.

Departamento Administrativo Nacional de Estadística (DANE). Página oficial del DANE [Internet]. Defunciones fetales. 2016; 2017 [citado el 21 de agosto de 2018]. Recuperado a partir de: https://www.dane.gov.co/index.php/estadisticas-portema/salud/nacimientos-y-defunciones/defunciones-fetales/defunciones-fetales-2016

Departamento Administrativo Nacional de Estadística (DANE). Página oficial del DANE [Internet]. Colombia - Índice de pobreza multidimensional - IPM - 2015; 2017 [citado el 14 de agosto de 2018] Recuperado a partir de: http://formularios.dane.gov.co/Anda_4_1/index.php/catalog/392

Fang, C., Liu, H., Li, G., Sun, D., \& Miao, Z. (2015). Estimating the impact of urbanization on air quality in China using spatial regression models. Sustainability, 7(11), 1557015592.

Giraldo, R., Caballero, W., \& Camacho-Tamayo, J. (2018). Mantel test for spatial functional data. AStA Advances in Statistical Analysis, 102(1), 21-39.

Jaramillo M. (2016). Situación de la mortalidad infantil en Colombia. [Tesis de Doctorado]. Granada: Universidad de Granada; 293 p.

Lurán, A., López, E., Pinilla, C., Sierra, P. (2009). Situación de la mortalidad por causas reducibles en menores de cinco años, Colombia, 1985-2004. Biomédica [revista en Internet]. [citado 2018 agosto 22]; 29 (1): 98-107.

Ministerio de Salud y Protección Social - Colciencias. Guía de práctica clínica del recién nacido prematuro. Guía No. 04. Centro Nacional de Investigación en Evidencia y Tecnologías en Salud CINETS; 2013. [citado el 21 de agosto de 2018]. Recuperado de:http://gpc.minsalud.gov.co/gpc_sites/Repositorio/Conv_500/GPC_rnp/gpc_rnp.asp $\mathrm{x}$.

Ministerio de Salud y Protección Social. Página Oficial del Ministerio de Salud y Protección Social [Internet]. En 2015 aumentó en 990.385 personas el número de afiliados al sistema de salud; 2016 [citado el 28 de agosto de 2018]. Recuperado a partir de: https://www.minsalud.gov.co/Paginas/En-2015-aumento-en-990.385-personas-el- 
numero-de-afiliados-al-sistema-de-salud.aspx

Ministerio de Salud y Protección Social. Análisis de situación de salud (ASIS) Colombia, 2016. Dirección de Epidemiología y Demografía; 2016 [citado el 29 de julio de 2018] Recuperado https://www.minsalud.gov.co/sites/rid/Lists/BibliotecaDigital/RIDE/VS/ED/PSP/asiscolombia-2016.pdf

Mogollón Pastrán, S. C. (2016) Mortalidad infantil: causas y determinantes sociales en municipios fronterizos de Colombia, estudio ecológico de análisis y tendencia. [Tesis de Doctorado]. Universidad Nacional de Colombia-Sede Bogotá, 119 p.

Ordoñez, K. A., \& Prieto, J. E. R. (2017). Estimación indirecta de la tasa de mortalidad infantil en Colombia, 1964-2008. Revista del Banco de la República, 87(1036), 13-38.

Organización Mundial de la Salud. Página oficial [Internet]. Nacimientos Prematuros; 2018 [citado el 15 de agosto de 2018] Recuperado a partir de: http://www.who.int/es/newsroom/fact-sheets/detail/preterm-birth

Ortiz, G. D., Navarro-Vargas, J. R., Eslava-Schmalbach, J. (2013) Inequidad en el sistema de salud: el panorama de la analgesia obstétrica. Revista Colombiana de Anestesiología, 41(3), 215-217.

Restrepo, N. (2017). La salud infantil en Colombia: un problema de inequidad. Rev. Médica Sanitas, 20(2), 64-66

Santacruz, J. S. R., \& Gil, C. A. D. (2013). Análisis espacial multitemporal de la tasa de mortalidad infantil municipal en Colombia (No. 012670). DEPARTAMENTO ADMINISTRATIVO NACIONAL DE ESTADISTICA-DANE.

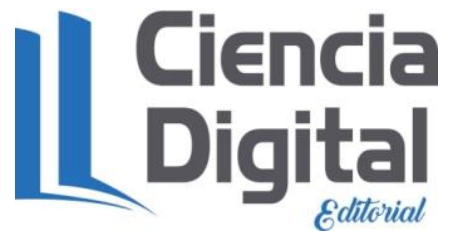


Para citar el artículo indexado.

Salcedo T. \& Ortiz A. (2018). Análisis espacial de la tasa de mortalidad infantil. Implementación de modelos de regresión espacial. Revista electrónica Ciencia Digital 2(4), 154-175.

http://cienciadigital.org/revistacienciadigital2/index.php/CienciaDigital/article/view/196/17 $\underline{4}$

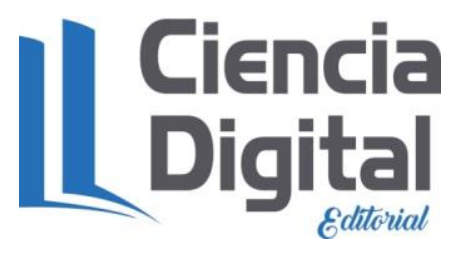

El artículo que se publica es de exclusiva responsabilidad de los autores y no necesariamente reflejan el pensamiento de la Revista Ciencia Digital.

El articulo queda en propiedad de la revista y, por tanto, su publicación parcial y/o total en otro medio tiene que ser autorizado por el director de la Revista Ciencia Digital.
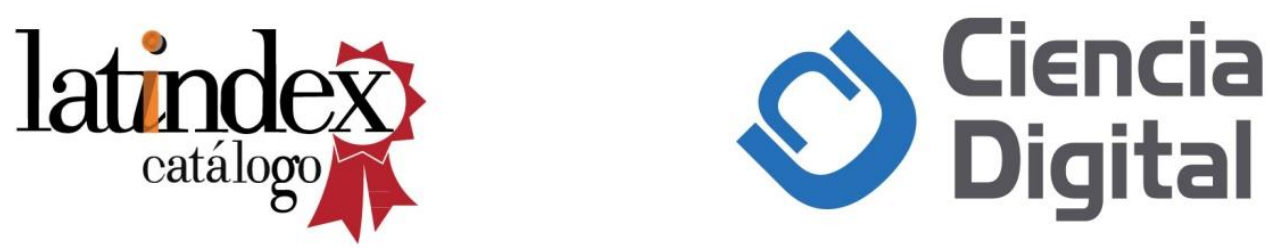